\title{
Investigating the Structure Preserving Encryption of High Efficiency Video Coding (HEVC)
}

\author{
Zafar Shahid and William Puech \\ Laboratory LIRMM, UMR CNRS 5506, University of Montpellier II \\ 161, rue Ada, 34392 MONTPELLIER Cedex 05, FRANCE \\ zafar.shahid@lirmm.fr, william.puech@lirmm.fr
}

\begin{abstract}
This paper presents a novel method for the real-time protection of new emerging High Efficiency Video Coding (HEVC) standard. Structure preserving selective encryption is being performed in CABAC entropy coding module of HEVC, which is significantly different from CABAC entropy coding of H.264/AVC. In CABAC of HEVC, exponential Golomb coding is replaced by truncated Rice (TR) up to a specific value for binarization of transform coefficients. Selective encryption is performed using AES cipher in cipher feedback mode on a plaintext of binstrings in a context aware manner. The encrypted bitstream has exactly the same bit-rate and is format complaint. Experimental evaluation and security analysis of the proposed algorithm is performed on several benchmark video sequences containing different combinations of motion, texture and objects.
\end{abstract}

\section{INTRODUCTION}

$\mathrm{HEVC}^{1}$ is the emerging video coding standard of ITU-T and ISO/IEC. HEVC achieves similar visual quality to its precedent H.264/AVC High Profile, with around 30\% bit-rate reduction for low delay mode, and with around 20\% bit-rate reduction for random access mode on average, but with lower complexity than H.264/AVC Baseline Profile. ${ }^{2}$ HEVC performs better because of some additional tools as summarized in Table 1.

It is pertinent to analyze this standard regarding its protection and authentication. Selective encryption (SE) is used to restrict access of video data to only authenticated users. For huge video data with real time constraints, SE encrypts a small part of the whole bit-stream with minimal resource overhead and provides sufficient protection for most of the applications. In this work, we have analyzed the selective encryption of HEVC in its CABAC entropy coding module, while fulfilling real-time constraints. Different encryption techniques including permutation, the Data Encryption Standard (DES) and the Advanced Encryption Standard (AES) ${ }^{3}$ have been used for the SE of video. SE of H.264/AVC has been studied in Ref. 4 wherein encryption has been carried out in some fields like intra-prediction mode, residual data, inter-prediction mode and motion vectors.

The use of general entropy coder as encryption cipher has been studied in the literature in Ref. 5. It encrypts nonzero coefficients by using different Huffman tables for each input symbols. The tables, as well as the order in which they are used, are kept secret. This technique is vulnerable to known plaintext attacks as explained in Ref. 6. Key-based interval splitting of arithmetic coding (KSAC) has used an approach ${ }^{7}$ where in intervals are partitioned in each iteration of arithmetic coding. Secret key is used to decide how the interval will be partitioned. Number of sub intervals in which an interval is divided should be kept small as it increases the bit rate of bitstream. Randomized arithmetic coding ${ }^{8}$ is aimed at arithmetic coding but instead of partitioning of intervals like in KSAC, secret key is used to scramble the order of intervals.

Yeung et al. proposed the perceptual video encryption at the transform stage by selecting one out of multiple unitary transforms. ${ }^{9}$ The unitary transforms were significantly different from discrete cosine transform (DCT) or discrete sine transform (DST), and the resulted coding efficiency is exactly the same to what can be achieved by using DCT or just falls very slightly. In Ref. 10, a quality assessment is performed for perceptual video encryption, wherein a user can still obtain some visible video contents (but at a very annoying quality) even without knowing the encryption key, is becoming more and more interesting in video applications such as video-on-demand $(\mathrm{VoD})$ and pay-TV. The main drawback of this scheme is that it needs a modification in the transform module of Codec, which is very unlikely for hardware codec chips and even for DSP codecs. Moreover, it is a challenge to keep all the transforms in the instruction cache, especially for embedded devices. 


\begin{tabular}{|c|c|}
\hline High Efficiency Configuration & Low Complexity Configuration \\
\hline \multicolumn{2}{|c|}{ Coding Unit tree structure (8x8 up to $64 \times 64$ luma samples) } \\
\hline \multicolumn{2}{|c|}{ Prediction Units } \\
\hline $\begin{array}{l}\text { Transform unit tree structure } \\
\text { (3 level max.) }\end{array}$ & $\begin{array}{c}\text { Transform unit tree structure } \\
\text { (2 level max.) }\end{array}$ \\
\hline \multicolumn{2}{|c|}{ Transform block size of $4 \times 4$ to $32 \times 32$ samples (always square) } \\
\hline \multicolumn{2}{|c|}{ Angular Intra Prediction (34 directions max.) } \\
\hline \multicolumn{2}{|c|}{ DCT-based interpolation filter for luma samples (1/4-sample, 8-tap) } \\
\hline \multicolumn{2}{|c|}{ DCT-based interpolation filter for luma samples (1/8-sample, 4-tap) } \\
\hline \multicolumn{2}{|c|}{ Coding Unit based Skip \& Prediction Unit based merging } \\
\hline \multicolumn{2}{|c|}{ Advanced motion vector prediction } \\
\hline \multicolumn{2}{|c|}{ CABAC } \\
\hline Internal bit-depth increase ( 4 bits) & $\mathrm{X}$ \\
\hline $\mathrm{X}$ & Transform precision extension (4 bits) \\
\hline \multicolumn{2}{|c|}{ Deblocking filter } \\
\hline Adaptive loop filter & $\mathrm{X}$ \\
\hline
\end{tabular}

Table 1: Tools for high efficiency and low complexity setting of HEVC.

Encrypted bitstream (EB) compliance is a required feature for multimedia applications and these techniques make the bitstream non-compliant and hence, cannot be decoded by standard H.264/AVC decoder. In Ref. 11, we have presented a selective encryption scheme of H.264/AVC based on CAVLC and CABAC entropy coding modules which fulfill realtime constraints by keeping the same bit rate and by generating completely compliant bitstream. Selective encryption of scalable extension of H.264/AVC has been proposed in literature by other researchers. ${ }^{12,13}$ In Ref. 14, Dubois et al. have proposed a format-compliant reduced selective encryption, wherein encryption ratio has been reduced while maintaining the minimum visual quality.

This paper is organized as follows: In Section 2, overview of CABAC entropy coding engine of HEVC is presented. It explains the working of CABAC of HEVC, its difference from CABAC of H.264/AVC regarding selective encryption. The proposed algorithm is presented in Section 3. Section 4 contains its experimental evaluation and performance analysis for benchmark video sequences and different QP values. In Section 5, we present the concluding remarks about the proposed scheme.

\section{CABAC ENTROPY CODING OF HEVC}

The development of HEVC was started with two entropy codings like H.264/AVC. First technique was based on variable length based entropy coding called low complexity entropy coding (LCEC). LCEC was aimed for low complexity, while having the same compression ratio as CAVLC. Second entropy coding technique was context adaptive binary arithmetic coding (CABAC), which is based on the principles of arithmetic coding. Later on, LCEC was removed from HEVC codec reference design. From HM-5.0 onward, LCEC entropy coding is removed from HEVC and CABAC is the only entropy coding technique available in HEVC. CABAC of HEVC has more sophisticated binarization stage as compared to CABAC of H.264/AVC. Moreover, binary arithmetic coding unit of CABAC of HEVC is implemented using tables to reduce the required processing power.

CABAC consists of binarization stage and binary arithmetic coding (BAC) stage as shown in Fig. 1. In the binarization step, non-binary syntax elements are converted to binary form called binstrings which are more amenable to compression by BAC. Binary representation for a non-binary syntax element is done in such a way that it is close to minimum redundancy code. In CABAC of HEVC, there are five basic code trees for binarization step, as compared to four basic binarization trees in CABAC of H.264/AVC as shown in Fig. 2. They are the unary code, the truncated unary code, the truncated rice code with context $\mathrm{p}(T R p)$, the kth order Exp-Golomb code (EGk) and the fixed length code. 


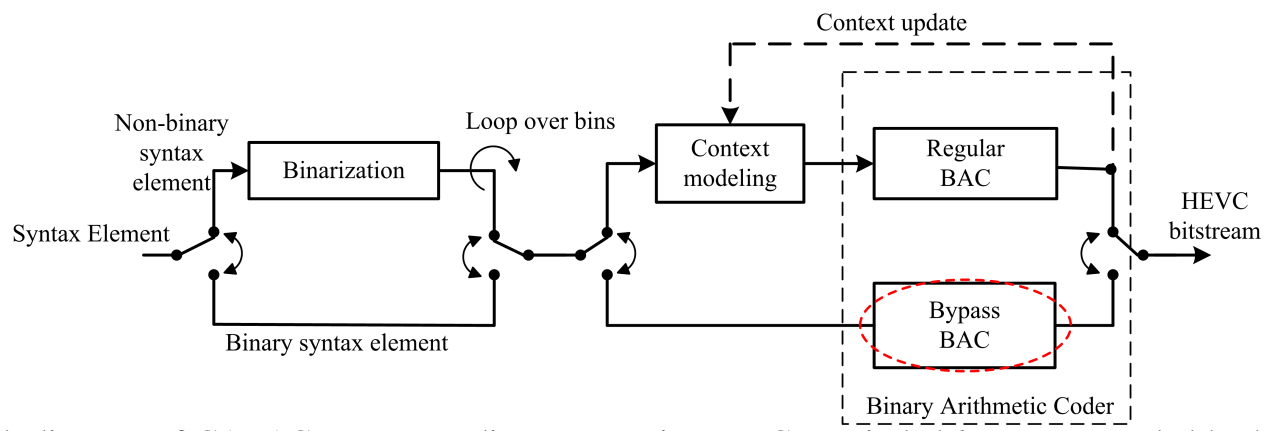

Figure 1: Block diagram of CABAC entropy coding process in HEVC. Encircled binstrings, coded by bypass-BAC are encrypt-able.

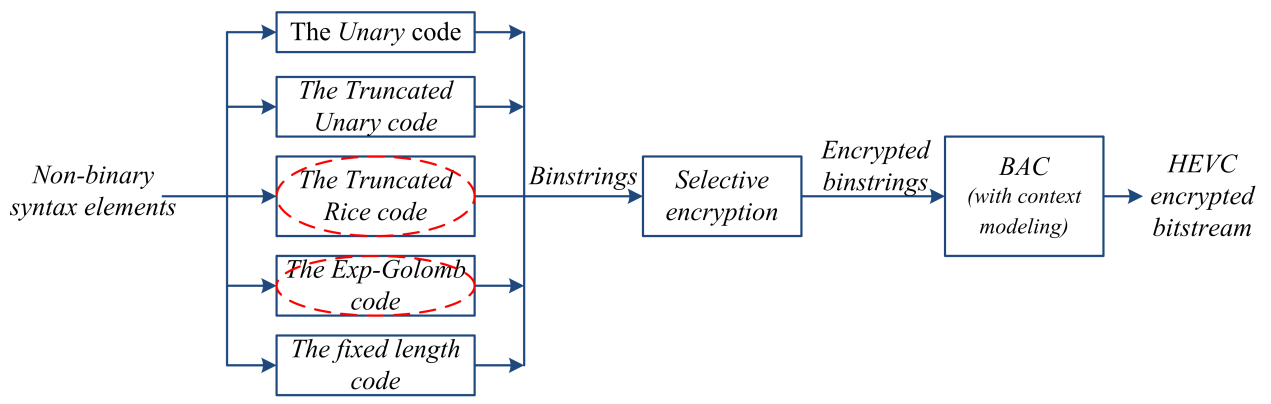

Figure 2: Selective encryption of CABAC-binstrings of HEVC. Encircled binarization techniques are encrypt-able.

For an unsigned integer value $x \geq 0$, the unary code consists of $x$ 1's plus a terminating 0 bit. The truncated unary code (TU) is only defined for $x$ with $0 \leq x \leq s$. For $x<s$, the code is given by the unary code, whereas for $x=s$ the terminating " 0 " bit is neglected.

The truncated rice code with context $p(T R p)$ is the newly introduced basic binary tree in HEVC for the first time. A $T R p$ binarization is a concatenation of quotient $(q)$ and remainder $(r)$ for a context $p$. For an unsigned integer value $x \geq 0$, the quotient $q$ is given by $q=\left\lfloor\frac{x}{p}\right\rfloor$ and the remainder $r$ is given by $r=x-q p$. For $p=0$ the TR0 binarization is exactly the TU binarization.

The EGk code is also a concatenation of a prefix and a suffix. For a given unsigned integer value $x>0$, the prefix part of the EGk binstring consists of a unary code corresponding to the length $l(x)=\left[\log _{2}\left(\frac{x}{2 k}+1\right)\right]$. The EGk suffix part is computed as the binary representation of $x+2^{k}\left(1-2^{l(x)}\right)$ using $k+l(x)$ significant bits. Consequently for EGk binarization, the code length is $2 l(x)+k+1$. When $\mathrm{k}=0$, the code length is $2 l(x)+1$. The fixed length code is applied to syntax elements with a nearly uniform distribution or to syntax elements, for which each bit in the fixed length code binstring represents a specific coding decision e.g., coded block flag.

In HEVC, quantized transform coefficients (QTCs) are binarized by concatenation of the basic code trees. Binarization of absolute level of QTCs is done by concatenation of the truncated rice code (TRp) and $E G 0$. Binarization and subsequent arithmetic coding process is applied to the syntax element coeff_abs_value_minus $3=$ abs_level -3 , since QTCs with zero magnitude are encoded using significant map.

\section{THE PROPOSED ALGORITHM}

Selectively encrypted HEVC bitstream will be format compliant and will fulfill real-time constraints provided the following conditions are fulfilled:

- Bit-rate constraint: the encrypted binstring must have the same length as the original binstring. 
- CABAC constraint: the encrypted binstring must be valid and decode-able by entropy decoder.

- Syntax element range constraint: the decoded value of syntax element from encrypted binstring must stay in the valid range for that syntax element. This is important for syntax elements which are used for prediction of neighboring blocks.

- Real-time constraint: Encryption space is defined as the number of valid values for some syntax element. The candidate binstrings should have a dyadic encryption space, which can be represented by integer number of bits. This is mandatory for real-time selective encryption, wherein a number of binstrings are concatenated to prepare a plaintext for AES encryption in CFB mode.

Format compliant SE cannot be performed on CABAC bitstream. Rather it is performed on binstrings which are input to BAC as shown in Fig. 2. Among all the five binarization techniques, the truncated rice with context $p$ (TRp) and EGk meet the conditions of selective encryption as highlighted in Fig. 2. The unary and truncated unary codes have different code lengths for each input value. They do not fulfill the first condition and their encryption will change the bit-rate of bitstream. In fixed length, different bits indicate different information regarding the header and is not viable for encryption. Suffix of $E G k$, and $T R p$ can be encrypted while keeping the bit-rate unchanged and both of them are used for binarization of QTCs.

\subsection{Encryption space}

From CABAC entropy engine point of view, we can encrypt only those binstrings which use bypass-BAC mode and use fixed contexts as highlighted in Fig. 1. Contexts for binstrings coded by regular-BAC are adaptive and their encryption make the bitstream non-format compliant because of mismatch of contexts on encoder and decoder side. All the syntax elements, which use bypass-BAC, are not encrypt-able. There are other conditions to be fulfilled e.g. same bit-rate, dyadic encryption space and same context if a context is used during binarization. For example, context $p$ is adaptive to QTC magnitude for $T R p$ binarization. To keep the bitstream format compliant, it is not allowed to modify the context $p$ for a specific QTC while performing selective encryption.

For binarization of QTCs, $E G k$ code is used in H.264/AVC. In HEVC, it is replaced with $T R p$ up to a specific value. ${ }^{15}$ The main motivation for using $T R p$ code is to increase the number of bypass bins by coding QTCs up to a specific maximum value with $T R p$. Moreover, $E G k$ code is optimally fit for distribution of H.264/AVC residuals, which is geometrical. While distribution of HEVC residuals is different and TRp code performs better compression for HEVC residuals.

Fig. 3 shows the encrypt-able suffixes for QTCs. Let us denote the QTCs by level in this section. TRp codes are used for binarization of levels up to a threshold given by:

$$
T R_{\text {limit }}[p]=\{7,14,26,46,78\} .
$$

If level is smaller than $T R \_$limit $[p]$ for $T R p$, it will be coded using $T R p$ codes and suffix of this $T R p$ code will be encrypted as shown in Fig. 3. Context $p$ for $T R p$ code is adaptive to value of level in the following manner:

$$
p_{\text {next }}[\text { level }]=\{3,5,12,24, \infty\}
$$

For example, let the current context $p$ is 0 . It will remain unchanged if level is 2 , but if will be modified to 1 if current level is 3. Context $p_{\text {next }}[$ level $]$ must remain unchanged during encryption of QTCs. Table 2 shows the TRp code values for contexts 0 and 1. TRp codes which cannot be used for real-time encryption are colored gray in this table. In a $T R p$ code with context $p$, we have $p$ encrypt-able bits except two limitations. First, $T R 0$ code is the same as truncated unary code and each binstring of $T R 0$ has different length and is not encrypt-able because of violation of bit-rate constraint. Second, the length of last equal-length group of $T R p$ codes is the same whether $E G 0$ code is appended or not as shown in Table 2. So, if we encrypt the binstrings of this group, it may make the bitstream non-complaint. To fulfill this constraint we have to reduce the encrypt-able bits for last equal length TR codes to half. More precisely, we can encrypt only $(p-1)$ LSBs of the suffix if MSB of the suffix is 0 . In Table 2, gray $T R p$ codes are not encrypt-able because of this limitation. 




Figure 3: Encryption process for levels and their signs in CABAC of HEVC.

\begin{tabular}{|l|lc|lc|c|}
\hline Symbol & TRcode[0] & Bits & TRcode[1] & Bits & $\ldots$ \\
\hline \hline 0 & 0 & 1 & 0 & 2 & \\
1 & 10 & 2 & 1 & 2 & \\
2 & 110 & 3 & 100 & 3 & \\
3 & 1110 & 4 & 101 & 3 & \\
4 & 11110 & 5 & 1100 & 4 & \\
5 & 111110 & 6 & 1101 & 4 & \\
6 & 1111110 & 7 & 11100 & 5 & \\
7 & 11111110 & 8 & 11101 & 5 & \\
8 & 11111111, EG0 & 9 & 111100 & 6 & \\
$\ldots$ & & & & & \\
18 & & & 11111111100 & 11 & \\
19 & & & 11111111101 & 11 & \\
20 & & & 11111111110 & 11 & \\
21 & & & 11111111111, EG0 & 12 & \\
$\ldots$ & & & & \\
\hline
\end{tabular}

Table 2: TRp code with context $p$. Gray cells are not encrypt-able.

If level is higher than a TR_limit $[p]$ limit, $E G 0$ code is used for the binarization of level - TR_limit $[p]$. In this case, suffix of $E G 0$ will be encrypted as shown in Fig. 3. It is important to note that $p_{n e x t}[$ level] constraint must be fulfilled even if $E G 0$ code is appended.

Along with the first three constraints for selective encryption, it is mandatory to fulfill the real-time constraint i.e. to ensure the dyadic encryption space which can be represented by integer number of bits. In case of QTCs, the threshold for context switch of $T R p$ code is not on a dyadic boundary as given in Eq. 1 and it may make the encryption space non-dyadic. Such type of encryption space can be encrypted separately, but it is not possible to put them in a plaintext for encryption by AES cipher in CFB mode.

\subsection{SE of HEVC in the CABAC entropy coding stage}

Let us consider $Y_{i}=X_{i} \oplus E_{k}\left(Y_{i-1}\right)$ as the notation for the encryption of a $n$ bit block $X_{i}$, using the secret key $k$ with the AES cipher in CFB mode. We have chosen to use this mode in order to keep the original compression rate. Indeed, with the CFB mode for each block, the size of the encrypted data $Y_{i}$ can be exactly the same one as the size of the plaintext $X_{i}$. In this mode, the code from the previously encrypted block is used to encrypt the current one. 
HEVC has introduced the concept of entropy slices. Context models are reset at the start of every entropy slice. Moreover, entropy slices restrict definition for neighborhood. Hence it is pertinent to perform SE of HEVC on each entropy slice independently. SE is performed on binstrings before compression by BAC. Non-binary syntax elements qre transformed to binstrings through process of binarization and at the same time we fill the $X_{i}$ with encrypt-able bits until either the vector $X_{i}$ is completely filled or the slice boundary arrives. Let $L\left(X_{i}\right)$ be the length up to which vector $X_{i}$ is filled. In case of slice boundary, if $L\left(X_{i}\right)<C$, we apply a padding function $p(j)=0$, where $j \in\left\{L\left(X_{i}\right)+1, \ldots, C\right\}$, to fill in the vector $X_{i}$ with zeros up to $C$ bits.

In the encryption step with AES in the CFB mode, the previous encrypted block $Y_{i-1}$ is used as the input of the AES algorithm in order to create $Z_{i}$. Then, the current plaintext $X_{i}$ is XORed with $Z_{i}$ in order to generate the encrypted text $Y_{i}$.

With the CFB mode of the AES algorithm, the generation of the encrypted stream $Z_{i}$ depends on the previous encrypted block $Y_{i-1}$. Consequently, if two plaintexts are identical $X_{i}=X_{j}$ in the CFB mode, then always the two corresponding encrypted blocks are different, $Y_{i} \neq Y_{j}$.

The decryption process in the CFB mode works in the same fashion except the fact that input is encrypted and output will be the plaintext. The decoded plaintext vector is split into segments in order to substitute the encrypted binstrings with original binstrings. The decoded binstrings substitute the encrypted binstrings in the bitstream. The bitstream is then further decoded to get the decrypted/original video frame using standard HEVC decoder steps.

\section{EXPERIMENTAL RESULTS}

In this section we analyze the results for the proposed selective encryption scheme for HEVC. We have used the reference implementation of HEVC HM 5.0 * for video sequences in HD resolution. For the experimental results, we have used the benchmark sequences of different resolution as shown in Table 3. This set of test material has been used in standardization process of HEVC. ${ }^{16}$

\begin{tabular}{|c|c|c|l|}
\hline Class & Res. & FPS & Videos \\
\hline \hline A & $2560 \times 1600$ & 30 & Traffic (S01), PeopleOnStreet (S02) \\
\hline B1 & $1920 \times 1080$ & 24 & Kimono1 (S03), ParkScene (S04) \\
\hline B2 & $1920 \times 1080$ & $50-60$ & Cactus (S05), BDrive (S06),BQTerrace (S07) \\
\hline C & $832 \times 480$ & $30-60$ & $\begin{array}{l}\text { BasketballDrill (S08), BQMall (S09), } \\
\text { PartyScene (S10), RaceHorses (S11) }\end{array}$ \\
\hline D & $416 \times 240$ & $30-60$ & $\begin{array}{l}\text { BPass (S12), BQSquare (S13), } \\
\text { BlowingBubbles (S14), RaceHorses (S15) }\end{array}$ \\
\hline E & $1280 \times 720$ & 60 & Vidyo1 (S16), Vidyo2 (S17), Vidyo3 (S18) \\
\hline
\end{tabular}

$*$ BDrive $=$ BasketballDrive, BPass $=$ BasketballPass

Table 3: Classification of benchmark video sequences used to evaluate the performance of the proposed SE technique.

To demonstrate the efficiency of our proposed scheme, we have compressed 50 frames with intra period of 10 . We have applied simultaneously our SE and HEVC compression as described in Section 3, on all the benchmark video sequences. We have analyzed the available encryption space (ES) and PSNR value of encrypted bitstream. ES is defined as percentage of encrypt-able bits in a video bitstream.

\subsection{Encryption space and PSNR analysis for benchmark video content}

Table 4 compares the average ES and PSNR of 50 frames of all benchmark video sequences at QP value 18 without encryption and with SE. Average PSNR value of luma for all the sequences at QP value 18 is $9.67 d B$ for SE. It confirms that this algorithm works well for various combinations of motion, texture and objects for intra and inter frames. Moreover, average PSNR values of $\mathrm{U}$ and $\mathrm{V}$ are $15.82 d B$ and $17.23 d B$ respectively, which are lower as compared to SE-CABAC

\footnotetext{
*https://hevc.hhi.fraunhofer.de/svn/svn_HEVCSoftware/
} 
of H.264/AVC values of $21.90 d B$ and $23.5 d B$ (Table IX of Ref. 11). Hence the proposed SE algorithm better protects the color information in HEVC video codec. Fig. 4 shows the visual quality of encrypted video frame for three different video sequences.

Table 4 also shows ES for SE for different benchmark video sequences for QP value 18, wherein ES varies from $14.83 \%$ to $17.76 \%$ based on the video content. Video sequences with static background (or with translational movement) like Vidyol have less ES. BQMall has also a lesser ES despite its complex background. It is due to the fact that background is static and is coded by skip blocks and translational motion in the foreground is very efficiently predicted. On the other hand, video sequences which have complex motion and moving background have high ES. For example, RaceHorses contains walking horses, camera movement and high- texture grass in the background and it has higher ES.



(a) S06 (1920x1080_50)

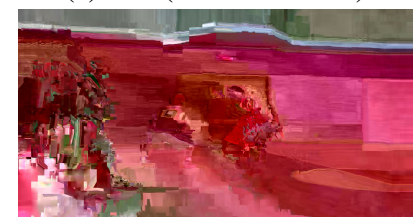

(d) SE (S06)

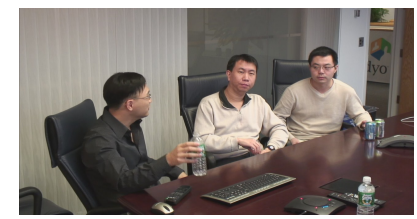

(b) S16 (1280x720_60)

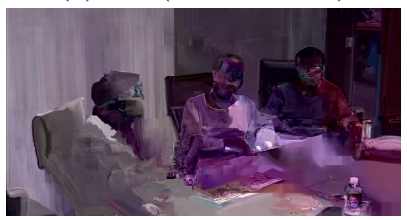

(e) SE (S16)



(c) S11 (832x480_30)

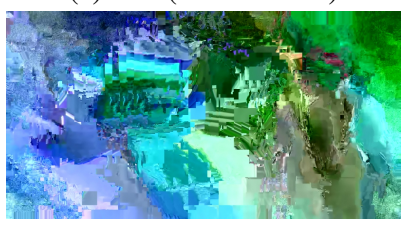

(f) SE (S11)

Figure 4: SE of different syntax elements for frame \# 8 of several video sequences having different resolution and frame rate at QP value 18: a-c) Encoded frames, d-f) Encoded and encrypted frames.

\subsection{Encryption space and PSNR analysis over whole range of QP values}

Table 5 compares the average PSNR of 50 frames of kimonol over whole range of QP values without encryption and with SE. One can note PSNR values of luma and chroma components remains in the lower range for all values of QP and the proposed SE is effective over whole range of QP values. Table 5 also provides analysis of the effect of QP on the ES for kimonol. One can note that ES decreases from $18.24 \%$ to $13 \%$ with increase in QP value. It is due to the fact that data part of video bitstream decreases with increase in QP value. The decrease in ES with increase in the QP value is very less for HEVC as compared to SE of CABAC of H.264/AVC wherein ES decreases from $19.97 \%$ to $9.46 \%$ (Table II of Ref. 11). It is because of the introduction of $T R p$ coding for binarization of QTCs.

\subsection{Security Analysis}

\subsubsection{Histogram analysis}

Histogram of the video frame gives the frequency distribution of the intensity levels or gray values. Here histograms of the original video frame and SE video frame are analyzed. Histogram graphs for original and SE video frame \#8 of kimono1 video sequence at QP value 18 are shown in Fig. 5. It is evident from Fig. 5.a and Fig. 5.b that both the histograms are entirely different. Moreover, histograms with 2 left and 2 right shifts in Fig. 5.c,d are also quite different.

\subsubsection{Encryption quality analysis}

The encryption quality (EQ) represents the average number of changes to each gray level $L .{ }^{17}$ Let $\mathrm{V}$ and $\mathrm{V}$ ' denote the original video frame and the encrypted video frame of a video sequence of resolution $m \times n$ with $L$ intensity levels. $V(x, y)$, $V^{\prime}(x, y) \in 0, . ., L-1$ are the gray levels of video frames $\mathrm{V}$ and $\mathrm{V}^{\prime}$ at position $(x, y)(0 \leq x \leq m-1,0 \leq y \leq n-1)$. Let $H_{L}(V)$ denote the number of occurrences of each gray level $L$ in the original frame $V$. Similarly, $H_{L}\left(V^{\prime}\right)$ denotes the number of occurrences of each gray level $L$ in encrypted frame $V^{\prime}$. The encryption quality is defined as:

$$
E Q=\frac{\sum_{L=0}^{255} H_{L}\left(V^{\prime}\right)-H_{L}(V)}{256}
$$




\begin{tabular}{|c|l|cc|cc|cc|}
\hline \multirow{2}{*}{ Seq } & ES & \multicolumn{2}{|c|}{ PSNR $(\mathrm{Y})(\mathrm{dB})$} & \multicolumn{2}{|c|}{ PSNR (U) (dB) } & \multicolumn{2}{c|}{ PSNR (V) (dB) } \\
& $\%$ & orig. & SE & orig. & SE & orig. & SE \\
\hline \hline S01 & 16.00 & 44.08 & 9.79 & 43.5 & 14.29 & 45.72 & 18.23 \\
S02 & 15.88 & 43.88 & 8.23 & 46.36 & 15.28 & 45.82 & 17.67 \\
\hline \hline S03 & 16.33 & 42.38 & 10.25 & 43.8 & 15.23 & 45.6 & 14.29 \\
S04 & 15.75 & 43.41 & 9.55 & 44.84 & 12.78 & 46.82 & 17.27 \\
S05 & 15.74 & 42.67 & 8.95 & 45.45 & 15.81 & 47.05 & 19.41 \\
S06 & 17.59 & 43.42 & 7.89 & 43.62 & 14.59 & 45.28 & 17.26 \\
S07 & 16.87 & 41.93 & 7.38 & 42.5 & 15.46 & 44.7 & 17.63 \\
\hline \hline S08 & 15.40 & 43.61 & 11.35 & 45.32 & 13.64 & 46.51 & 11.84 \\
S09 & 14.83 & 42.21 & 10.86 & 44.55 & 18.48 & 46.37 & 16.73 \\
S10 & 16.98 & 43.04 & 8.99 & 43.86 & 16.13 & 44.55 & 20.23 \\
S11 & 17.76 & 43.17 & 10.64 & 43.95 & 11.22 & 44.68 & 14.48 \\
\hline \hline S12 & 15.69 & 44.66 & 13.76 & 46.61 & 23.46 & 46.66 & 14.39 \\
S13 & 16.67 & 42.39 & 6.72 & 45.53 & 22.2 & 46.22 & 19.19 \\
S14 & 15.98 & 41.92 & 12.15 & 43.39 & 12.04 & 45.21 & 22.22 \\
S15 & 15.73 & 42.94 & 8.75 & 44.01 & 16.75 & 44.45 & 16.25 \\
\hline \hline S16 & 15.42 & 45.35 & 10.32 & 47.68 & 15.23 & 48.78 & 18.95 \\
S17 & 16.01 & 44.86 & 9.42 & 49.23 & 14.56 & 49.03 & 17.79 \\
S18 & 15.45 & 45.35 & 8.98 & 49.34 & 17.54 & 49.61 & 16.39 \\
\hline avg. & 16.11 & 43.4 & 9.67 & 45.2 & 15.82 & 46.28 & 17.23 \\
\hline
\end{tabular}

Table 4: Encryption space and PSNR analysis of benchmark video sequences at QP value 18.

\begin{tabular}{|c|c|cc|cc|cc|}
\hline QP & \multirow{2}{*}{$\begin{array}{c}\text { ES } \\
\end{array}$} & \multicolumn{2}{|c|}{ PSNR $(\%)$} & $(\mathrm{dB})$ & \multicolumn{2}{|c|}{ PSNR $(\mathrm{U})(\mathrm{dB})$} & \multicolumn{2}{c|}{ PSNR $(\mathrm{V})(\mathrm{dB})$} \\
\cline { 3 - 8 } & & orig. & SE & orig. & SE & orig. & SE \\
\hline \hline 12 & 18.24 & 47.67 & 9.12 & 48.23 & 13.03 & 49.62 & 10.77 \\
18 & 16.33 & 43.37 & 9.55 & 44.80 & 12.78 & 46.83 & 17.27 \\
24 & 16.19 & 41.47 & 9.91 & 42.69 & 17.42 & 44.39 & 13.32 \\
30 & 15.56 & 38.89 & 8.15 & 41.02 & 17.46 & 42.53 & 17.31 \\
36 & 14.91 & 35.79 & 10.18 & 39.71 & 16.60 & 41.22 & 13.48 \\
42 & 13.00 & 32.66 & 12.35 & 38.80 & 19.91 & 40.50 & 17.31 \\
\hline
\end{tabular}

Table 5: Encryption space and PSNR analysis for kimono1 video sequence over whole range of QP values.

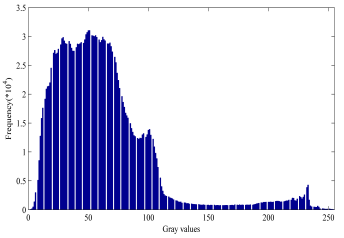

(a) Original

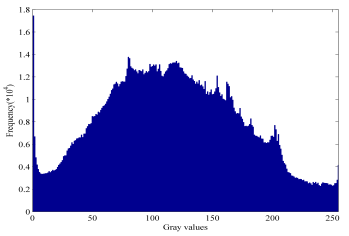

(b) SE frame (no shift)

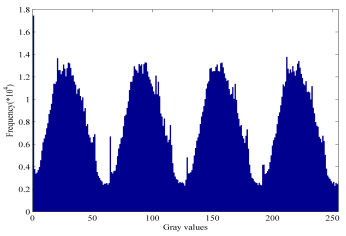

(c) SE frame (2 left shift)

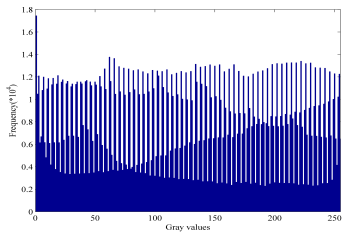

(d) SE frame (2 right shift)

Figure 5: Histogram analysis of original and SE video frame \#8 of kimono video sequence at QP value 18.

The encryption quality for frame \# 8 of kimono is shown for QP value of 18 in Table 6 for different number of shifts, wherein encryption quality remains in the higher range for all the shifts.

\begin{tabular}{|c|cccccccc|}
\hline left bit shift & 0 & 1 & 2 & 3 & 4 & 5 & 6 & 7 \\
\hline EQ & 7002 & 6955 & 7100 & 6979 & 6879 & 7102 & 7199 & 6989 \\
\hline
\end{tabular}

Table 6: Encryption quality for SE of kimono at QP value 18. 


\subsubsection{Sensitivity analysis}

To provide an encryption algorithm with high security, cryptosystem should be highly sensitive towards the key to make any brute-force attack ineffective. The ciphertext should not be decrypted correctly although there is only a slight difference between encryption or decryption keys. For this purpose, a key sensitivity test is assumed where we pick one key and then applied the proposed technique for encryption and then make one bit change in the key and decode the bitstream. Numerical results show that the proposed technique is highly sensitive towards the key change, i.e., the video frame decrypted with slightly different key is just another encrypted video frame, as shown in Fig. 6.

\subsubsection{Approximation attack}

If the syntax, context and statistical information is known a priori then the encrypted multimedia content may be approximately recovered even if the encrypted part is provably secure. To verify the ability of the proposed technique against approximation attack, it is assumed that an intruder knows the encrypted suffices of the binstrings, but not the bit values. These bit locations are then set to 0 . An approximate copy of the original content is obtained by reconstructing the video frame with this partial information. Fig. 7 shows frame \#0 of kimono video sequence with QP value 24 obtained with this partially assumed data. It is evident that quality of the decoded video frame is even worse than encrypted frame. For example, Fig. 7 shows that luma of attacked SE video frame has $P S N R=5.45 d B$ as shown in Fig. 7.

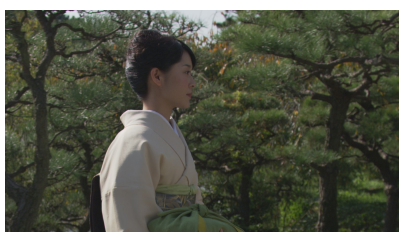

(a)

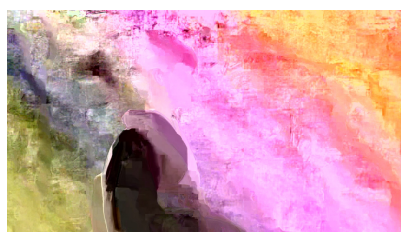

(b)

Figure 6: Key sensitivity test for kimono \#0. SE frame is decrypted with: a) Original key: PSNR (YUV) $=\{45.78,47.04,48.88\} \mathrm{dB}$, b) 1-bit different key: PSNR (YUV) $=\{9.45,19.11,13.63\} \mathrm{dB}$.

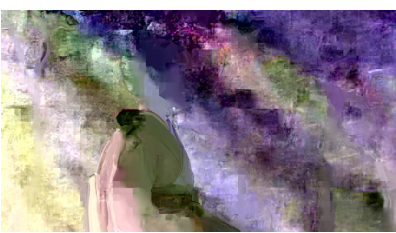

(a)

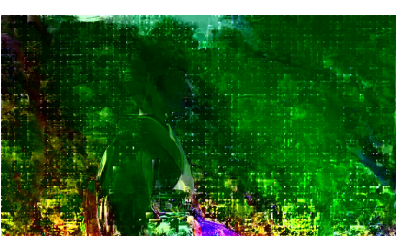

(b)
Figure 7: Known plaintext attack for kimono \#0: a) Encrypted frame: PSNR (YUV) $=\{10.12,20.65,14.23\} \mathrm{dB}, \mathrm{b})$ Approximation attacked frame: PSNR $(Y U V)=\{11.12,20.19,14.69\} \mathrm{dB}$.

\section{CONCLUSION}

In this paper, an efficient SE system has been proposed for HEVC video codec for its CABAC entropy coding. The security analysis verifies that the proposed scheme offer enough protection against crypt-analysis attacks. The SE is performed on the entropy slices independently in HEVC. In this way the proposed encryption method does not affect the parallelism of HEVC. Moreover, the proposed technique does not change bit-rate and the HEVC bitstream compliance. The SE is performed in CABAC binstrings such that they remain a valid binstrings having exactly the same length. The proposed method has the advantage of being suitable for streaming over heterogeneous networks because of no change in bit-rate. The experiments have shown that we can achieve the desired level of encryption under a minimal set of computational requirements.

\section{REFERENCES}

[1] HEVC, "High Efficiency Video Coding (HEVC) Text Specification Draft 6," tech. rep., ITU-T/ISO/IEC Joint Collaborative Team on Video Coding (JCT-VC), Doc. JCTVC-H1003, Geneva, CH (November 2011).

[2] Ugur, K., Andersson, K., and Fuldseth, A., "Video Coding Technology Proposal by Tandberg, Nokia, and Ericsson," tech. rep., Joint Video Team (JVT), JCTVC-A119, Dresden, Germany (April 2010).

[3] Uhl, A. and Pommer, A., [Image and Video Encryption: From Digital Rights Management to Secured Personal Communication], Springer (2005).

[4] Lian, S., Liu, Z., Ren, Z., and Wang, Z., "Selective Video Encryption Based on Advanced Video Coding," Lecture notes in Computer Science, Springer-verlag (3768), 281-290 (2005).

[5] Wu, C.-P. and Kuo, C.-C., "Design of Integrated Multimedia Compression and Encryption Systems," IEEE Transactions on Multimedia 7, 828-839 (2005).

[6] Jakimoski, G. and Subbalakshmi, K., "Cryptanalysis of Some Multimedia Encryption Schemes," IEEE Transactions on Multimedia 10, 330-338 (April 2008). 
[7] Jiangtao, W., Hyungjin, K., and Villasenor, J., "Binary arithmetic coding with key-based interval splitting," IEEE Signal Processing Letters 13, 69-72 (Feb. 2006).

[8] Grangetto, M., Magli, E., and Olmo, G., "Multimedia Selective Encryption by Means of Randomized Arithmetic Coding," IEEE Transactions on Multimedia 8, 905-917 (October 2006).

[9] Yeung, S. K. A., Zhu, S., and Zeng, B., "Design of New Unitary Transforms for Perceptual Video Encryption," IEEE Transactions on Circuits and Systems for Video Technology 21, 1341 -1345 (September 2011).

[10] Yeung, S. K. A., Zhu, S., and Zeng, B., "Quality Assessment for a Perceptual Video Encryption System," in [Proc. IEEE International Conference on Wireless Communications, Networking and Information Security], 102 -106 (June 2010).

[11] Shahid, Z., Chaumont, M., and Puech, W., "Fast Protection of H.264/AVC by Selective Encryption of CAVLC and CABAC for I \& P Frame," IEEE Transactions on Circuits and Systems for Video Technology 21, 565-576 (May 2010).

[12] Asghar, M. and Ghanbari, M., "An Efficient Security System for CABAC Bin-strings of H.264/SVC," IEEE Transactions on Circuits and Systems for Video Technology (99) (2012).

[13] Stutz, T. and Uhl, A., "A Survey of H.264 AVC/SVC Encryption," IEEE Transactions on Circuits and Systems for Video Technology 22, 325 -339 (March 2012).

[14] Dubois, L., Puech, W., and Blanc-Talon, J., "Fast Protection of H.264/AVC by Reduced Selective Encryption of CAVLC," in [Proc. European Signal Processing Conference], 2185-2189 (2011).

[15] Nguyen, T., "CE11: Coding of transform coefficient levels with Golomb-Rice codes," tech. rep., ITU-T/ISO/IEC Joint Collaborative Team on Video Coding (JCT-VC), Doc. JCTVC-E253, Geneva, CH (March 16-23 2011).

[16] Baroncini, V., Ohm, J.-R., and Sullivan, G., "Report of Subjective Test Results of Responses to the Joint Call for Proposals (CfP) on Video Coding Technology for High Efficiency Video Coding (HEVC)," tech. rep., ITU-T/ISO/IEC Joint Collaborative Team on Video Coding (JCT-VC), Doc. JCTVC-A204, Geneva, CH (April 15-23 2010).

[17] Ahmed, H., Kalash, H., and Allah, O., "Encryption Efficiency Analysis and Security Evaluation of RC6 Block Cipher for Digital Images," in [Proc. International Conference on Electrical Engineering], 1 -7 (April 2007). 